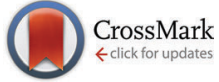

Cite this: DOI: $10.1039 / \mathrm{c} 5 \mathrm{mb} 00034 \mathrm{c}$

\title{
Proteomic analysis of human sonic hedgehog (SHH) medulloblastoma stem-like cells $\dagger$
}

\author{
Maurizio Ronci, $\ddagger^{\text {abc }}$ Giuseppina Catanzaro, $\ddagger^{d}$ Luisa Pieroni, ${ }^{\text {be }}$ Agnese Po, ${ }^{d}$ \\ Zein Mersini Besharat, ${ }^{d}$ Viviana Greco, ${ }^{\text {be }}$ Stefano Levi Mortera, be Isabella Screpanti, df \\ Elisabetta Ferretti*d and Andrea Urbani*be
}

\begin{abstract}
Human medulloblastoma (MB) is a malignant brain tumor that comprises four distinct molecular subgroups including the Sonic Hedgehog (SHH)-MB group. A leading cause of the SHH subgroup is an aberrant activation of the $\mathrm{SHH}$ pathway, a developmental signaling that regulates postnatal development of the cerebellum by promoting the mitotic expansion of granule neural precursors (GNPs) in the external granule layer (EGL). The abnormal SHH signaling pathway drives not only SHH-MB but also its cancer stem-like cells (SLCS), which represent a fraction of the tumor cell population that maintain cancer growth and have been associated with high grade tumors. Here, we report the first proteomic analysis of human SHH-MB SLCs before and after Retinoic Acid (RA)-induced differentiation. A total of 994 nLC-MS buckets were statistically analysed returning 68 modulated proteins between SLCs and their differentiated counterparts. Heat Shock Protein 70 (Hsp70) was one of the proteins that characterized the protein profile of SLCs. By means of Ingenuity Pathway Analysis (IPA), Genomatix analysis and extending the network obtained using the differentially expressed proteins we found a correlation between Hsp70 and the NF- $\mathrm{KB}$ complex. A key driver of the SHH-MB group is CMET whose downstream proliferation/survival signalling is indeed via PI3K/Akt/NF-KB. We confirmed the results of the proteomic analysis by western blot, underlining that a P-p65/NF- $\mathrm{kB}$ activatory complex is highly expressed in SLCs. Taking together these results we define a new protein feature of SHH-MB SLCs.
\end{abstract}

www.rsc.org/molecularbiosystems

Received 12th January 2015, Accepted 26th February 2015

DOI: $10.1039 / \mathrm{c} 5 \mathrm{mb} 00034 \mathrm{c}$

\section{Introduction}

Medulloblastoma (MB) is a malignant brain tumor occurring in childhood and adults. ${ }^{1}$ Despite multimodal therapy, MB can recur and survivors often develop severe long-term neurological side effects, which have prompted efforts to develop new therapeutic strategies. ${ }^{2}$ Based on the key developmental signalling pathways, which regulate $\mathrm{MB}$ cells, in recent years high-throughput studies ${ }^{3-10}$ allowed us to classify MB into four subgroups (WNT-wingless,

\footnotetext{
${ }^{a}$ Department of Medical, Oral and Biotechnological Sciences,

University G. d'Annunzio of Chieti-Pescara, Chieti, Italy

${ }^{b}$ Santa Lucia IRCCS Foundation, Rome, Italy

${ }^{c}$ Mawson Institute, University of South Australia, Mawson Lakes, SA, 5095 Australia

${ }^{d}$ Departments of Molecular Medicine and Experimental Medicine University of Sapienza, Rome, Italy.E-mail: elisabetta.ferretti@uniroma1.it; Fax: +39 0649255660; Tel: +390649255135

${ }^{e}$ Department of Experimental Medicine and Surgery, University of Rome

"Tor Vergata", Rome, Italy. E-mail: andrea.urbani@uniroma2.it;

Fax: +39 0650170 3222; Tel: +3906 501703215

${ }^{f}$ Center for Life NanoScience@Sapienza, Istituto Italiano di Tecnologia, Rome, Italy $\dagger$ Electronic supplementary information (ESI) available. See DOI: 10.1039/ c5mb00034c

\# These authors have contributed equally to the manuscript.
}

SHH-Sonic Hedgehog, Group 3 and Group 4) characterized by distinct mutations as well as different clinical outcomes. ${ }^{1,6,10,11}$ The heterogeneity of MB subgroups parallels their recently identified distinct cells of origin, whose aberrant development is responsible for tumorigenesis. ${ }^{7,12}$

In normal development, Hedgehog $(\mathrm{HH})$ functions as a morphogen to induce cell identities in the ventral spinal cord and as a mitogen to drive proliferation of granule neural precursors (GNPs), ${ }^{12}$ a neuronal cell population that undergoes proliferation in the external granule layer (EGL) followed by migration in the internal granule layer (IGL) and differentiation into mature granules during cerebellar development. ${ }^{13}$ Activation of the SHH pathway starts with the interaction of the ligand with the inhibitory receptor Patched1 (Ptch1), which causes the depression of the transmembrane transducer Smoothened (Smo). Following ligand/receptor interaction, Smo migrates to the top of the cilium, a microtubule based organelle, and triggers a series of intracellular events that terminates with the activation of the Gli transcription factors (Gli1, Gli2 and Gli3). The transcriptional targets regulated by the $\mathrm{SHH} / \mathrm{Gli}$ pathway have been in part elucidated and include genes involved in key cellular processes such as the cell cycle, survival, migration and metabolism. $^{14}$ 
$\mathrm{HH}$ abnormal activation in GNPs leads to the development of one of the four MB subtypes, SHH-MB. ${ }^{15}$ SHH-MBs are the most common $\mathrm{MB}$ types in infants and adults, whereas in children other subgroups are more frequent. ${ }^{11}$ SHH-MBs in infants, children, and adults differ in transcriptome, methylome, and in the number and type of mutations constituting a very heterogeneous group of tumors. ${ }^{16}$ A very recent study showed that SHH-MBs with tumor relapse and poor survival are characterized by active MET kinase signaling. ${ }^{17}$

Stem cells or cancer stem-like cells (SLCs) represent a fraction of the tumor cell population which are not only the progenitors that give rise to $\mathrm{MB}^{12}$ and sustain cancer growth, but have also been associated with poor prognosis in several kinds of tumors ${ }^{18,19}$ by driving both resistance to therapy and disease relapse. ${ }^{12,20}$ Molecular characterization of human malignant brain tumors, and in particular SLCs, has identified a broad spectrum of genes and molecular mechanisms inhibiting neuronal and glial differentiation. ${ }^{21}$

We previously described SLCs derived from human SHH-MBs. ${ }^{22}$ Since a thorough understanding of the molecular pathways sustaining MB SLCs is mandatory to design novel specific therapeutic strategies, we decided to investigate proteome features of SHH-MB SLCs and their retinoic acid (RA) differentiated counterparts applying a novel label-free quantitative proteomic analysis based on a double stage approach to maximize the identification capacities of the statistically differential spectral features. Such a technological workflow is different from the already employed data-independent differential profiling, which is well assessed and described. ${ }^{23,24}$ In fact the two steps allow the increase in the identification capability of MS/MS data by applying a data dependent acquisition approach on a limited number of significant spectral features.

\section{Results}

\section{Label free differential proteomics of SHH-MB SLCs}

Initial studies were performed to evaluate whether the SLCs could be differentiated through the use of RA treatment. After $48 \mathrm{~h}$ of treatment, we were able to detect the presence of neuronal and glial markers $\beta 3$-Tubulin and Glial Fibrillary Acidic Protein (GFAP) respectively. Protein expression of these markers was confirmed both through western blot and immunofluorescence (Fig. 1A and B).

The statistical analysis of the molecular features (accurate mass-retention time pairs) extracted from 4 instrumental replicates per condition returned 518 significantly different target signals at $p<0.05$ out of the 944 total reproducible spectral features included in the statistical evaluation. As shown in the volcano plot in Fig. 2A and Table S1 (ESI $\dagger$ ) they result evenly distributed between up and down regulated.

Two untargeted MS/MS acquisitions were imported in ProteinScape together with the Scheduled Precursor List (SPL) acquisitions and the quantitation tables. Database search was performed and the results were merged in a single virtual sample.

Proteins whose expression in RA-treated cells versus SLCs was more than 1.2 times or less than 0.8 fold regulated and that

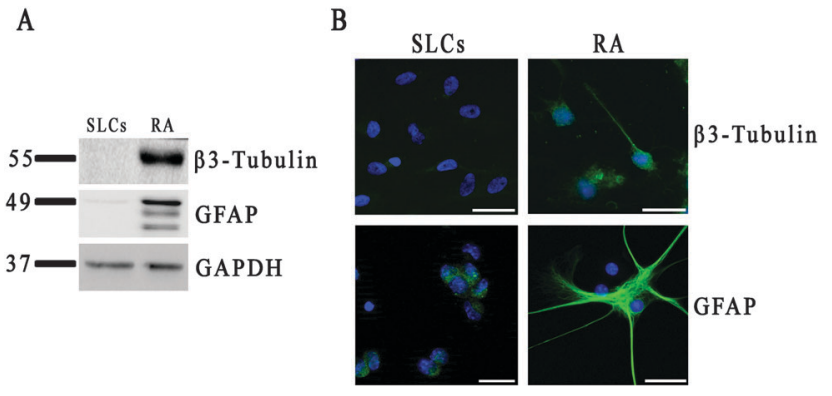

Fig. 1 SHH-MB SLCs before and after RA-induced differentiation. Western blot $(A)$ and immunofluorescence $(B)$ analyses of neuronal ( $\beta 3$-Tubulin) and glial (Glial Fibrillary Acidic Protein, GFAP) markers in SLCs and RA-treated cells. Exposure to RA for $48 \mathrm{~h}$ induced a strong increase in both differentiation markers. In (A) Gliceraldehyde 3-Phosphate Dehydrogenase (GAPDH) was used as an internal control. In (B) magnification $40 \times$, bars $5 \mu \mathrm{m}$.
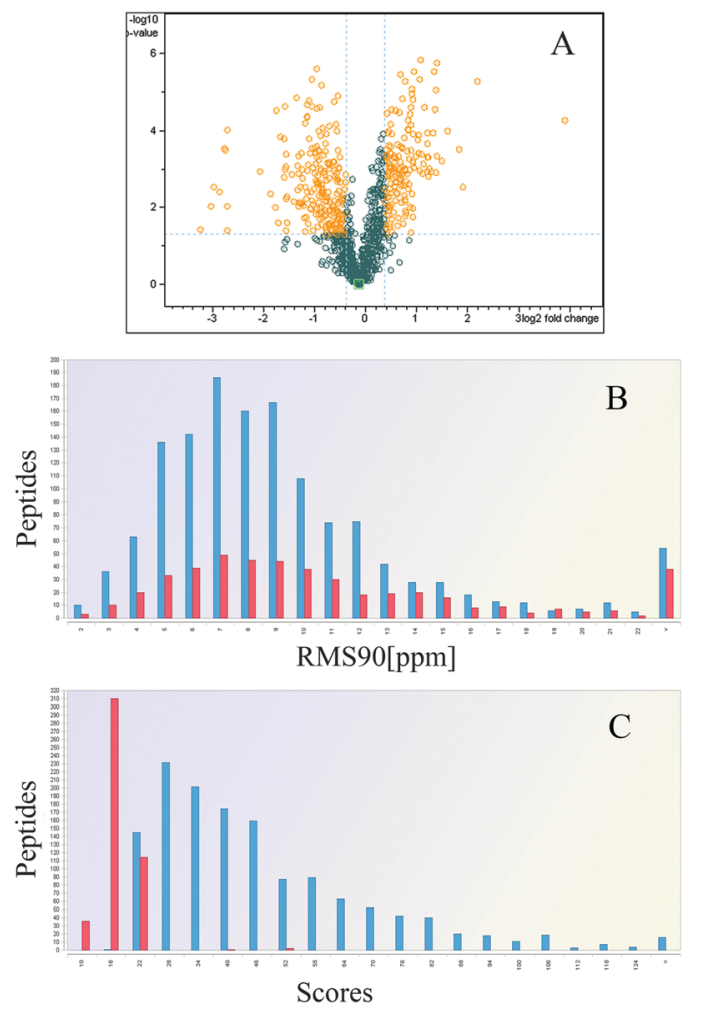

Fig. 2 (A) Volcano plot showing the distribution of significantly differential signals detected in the MS only chromatographic runs; thresholds are set to $p<0.05$ and a fold change limit of 1.3. (B) Peptide identification statistics RMS90 [ppm] error distribution, accepted in blue bars and not accepted in red bars, centered around 6 ppm. (C) Mascot score distribution of the identified peptides.

showed $p<0.05$ in the difference in the single peptide resulting in 68 were considered for further processing (Table S2, ESI $\dagger$ ). Data quality was evaluated by checking the analytical performances in the experimental dataset. In Fig. $2 \mathrm{~B}$ and $\mathrm{C}$ the distribution of the 'deviation from predicted mass' at $90 \%$ confidence interval in ppm (RMS90) of accepted identifications (blue) paired to the identifications that were not accepted (red) and the distribution of the Mascot score for protein identification (accepted in blue bars and 
not accepted in red bars) are reported. FDR was set to be less than $1 \%$ from the Mascot search engine results.

A manual and preliminary inspection of the protein data set confirmed some known evidence and therefore the consistency of the label free shotgun analysis. For example GFAP_HUMAN, which is an accepted marker of differentiation, was present only in RA-treated cells and G3P_HUMAN Glyceraldehyde 3-Phosphate Dehydrogenase (GAPDH), which is known not to vary with differentiation, resulted not modulated.

\section{Identification of protein networks associated with SHH-MB SLCs}

The identified proteins differentially expressed in SHH-MB SLCs before and after RA-treatment were used for enrichment analysis. Tools available at Webgestalt were exploited to obtain biological insights from the list. The KEGG enrichment analysis showed that the metabolic pathway (hsa01100; $\operatorname{adj} P=4.52 \times$ $\left.10^{-11}\right)$, protein processing in the endoplasmic reticulum pathway (hsa04141; $\operatorname{adj} P=3.86 \times 10^{-13}$ ) and glycolysis/gluconeogenesis pathways (hsa00010; $\operatorname{adj} P=2.25 \times 10^{-8}$ ) were among the most significantly represented (Table S3, ESI $\dagger$ ). Histograms showing the results of the GO slim classification tool of Webgestalt Molecular functions, biological processes and cellular components are presented in Fig. S1 (ESI $\dagger$ ). Interestingly the most represented ontology in the category biological processes is the metabolic process.

The list of differential proteins was also subjected to Bioprofiling. Fig. S2 (ESI $\dagger)$ shows a significant network $(p<0.005)$ model generated by the protein-protein Interaction (PPI) spider tool that analyzes the gene/protein list using as reference the knowledge of physical associations and direct interactions from the IntAct database. For the human genome, the reference network covers about 7960 genes involved in approximately 40000 unique pairwise interactions. The model returns referenced interactions of the proteins in the dataset allowing 1 missing gene. The resulting model includes 71 interactions. Database evidence for each interaction is reported in Table S4 in the ESI. $\dagger$

The proteins resulted significantly deregulated were subsequently subjected to pathway analysis using Ingenuity Pathways Analysis (IPA) in order to assign them to different functional networks. The top canonical pathways represented in the dataset resulted in the 14-3-3-mediated signaling, the Remodeling of Epithelial Adherens Junctions, the Glycolysis I, and Gluconeogenesis I. The activation $Z$-score predicted among the 'Disease and Bio Functions' that 'cell death of neuroblastoma cell lines' and 'cell death of tumor cell lines' are decreased (Table 1).

Furthermore, highly significant networks resulting from IPA (Fig. 3 and Table 2, Fig. S3, ESI $\dagger$ ) showed that the NF- $\kappa$ B complex and p53 are the pivotal players. We selected the potential biological pathway associated with Hedgehog driven cancers and stemness networks.

In order to endorse the IPA, we generated a network using the Genomatix Pathway System (GePS), including differentially expressed proteins as input (Fig. S4, ESI $\dagger$ ). To better evaluate the connections among pathways highlighted by IPA, we included transcriptional downstream targets of differentially expressed proteins in the network. Since tumor cells are cells that are stressed by a series of stimuli such as hypoxia and oxidative stress, we focused our attention on Heat Shock Protein 70 (Hsp70) that resulted over expressed in SLCs. Analysing the network reported in Fig. 3 we noticed that Hsp70 is connected to Akt rather than p53. However, since Akt is an upstream regulator of $\mathrm{NF}-\kappa \mathrm{B}$, which is a hub protein in the second significantly represented network, we decided to further investigate this important complex. Therefore we performed validation experiments by western blot.

\section{Agreement of differential profiling between commercial antibodies and MS data}

To evaluate the agreement of the potential results achievable with commercial non-qualified affinity reagents and the specific differential MS analysis, we selected some biologically relevant proteins and by means of western blot analysis, we evaluated their abundance in SHH-SLCs and after differentiation (Fig. 4). The results confirm good agreement between the two methodologies.

Among the functional networks derived from IPA, we analysed in depth the one connected with the cell death of cancer cells and we found that hub proteins in this network are mitogen-activated protein kinase P38 and NF- $\kappa \mathrm{B}$.

\section{P-p65/NF-кB activatory complexes are highly expressed in SHH-MB SLCs}

Since NF-кB was one of the central nodes in the network analysis, we further investigated proteins of the NF- $\mathrm{B}$ pathway by western blot (Fig. 5). We firstly analyzed IKB $\alpha$, the principal inhibitory protein of the NF- $\mathrm{B}$ complex, whose phosphorylation results in ubiquitination, dissociation of IKB $\alpha$ from NF- $\mathrm{BB}$, and eventual degradation of $\mathrm{IKB} \alpha$ by the proteosome followed by nuclear translocation of the

Table 1 IPA results showing the predicted activation Z-score and the molecules involved in the 'Disease and Bio Functions'

\begin{tabular}{|c|c|c|c|c|c|c|}
\hline Categories & $\begin{array}{l}\text { Diseases or } \\
\text { functions } \\
\text { annotation }\end{array}$ & $p$-value & $\begin{array}{l}\text { Predicted } \\
\text { activation } \\
\text { state }\end{array}$ & $\begin{array}{l}\text { Activation } \\
Z \text {-score }\end{array}$ & Molecules & \# Molecules \\
\hline $\begin{array}{l}\text { Cell death } \\
\text { and } \\
\text { survival }\end{array}$ & $\begin{array}{l}\text { Cell death of } \\
\text { neuroblastoma } \\
\text { cell lines }\end{array}$ & $3.24 \times 10^{-11}$ & Decreased & -2.897 & $\begin{array}{l}\text { ATP5A1, CCT2, CCT3, CCT5, CCT7, CCT8, ENO1, GAPDH, } \\
\text { HSPA1A/HSPA1B, HSPA8, TCP1, TUBA1A }\end{array}$ & 12 \\
\hline $\begin{array}{l}\text { Cell death } \\
\text { and } \\
\text { survival }\end{array}$ & $\begin{array}{l}\text { Cell death of } \\
\text { tumor cell lines }\end{array}$ & $2.40 \times 10^{-12}$ & Decreased & -2.178 & $\begin{array}{l}\text { ALB, ATP5A1, CCT2, CCT3, CCT5, CCT7, CCT8, DTYMK, ENO1, } \\
\text { FAU, GAPDH, HINT1, HNRNPC, HNRNPK, HSP90AB1, HSPA1A/ } \\
\text { HSPA1B, HSPA5, HSPA8, HSPA9, LGALS1, LMNA, NPM1, PCNA, } \\
\text { PKM, PPIA, PRDX1, TCP1, TUBA1A, TUBB3, VCP, XRCC6, YWHAZ }\end{array}$ & 32 \\
\hline
\end{tabular}




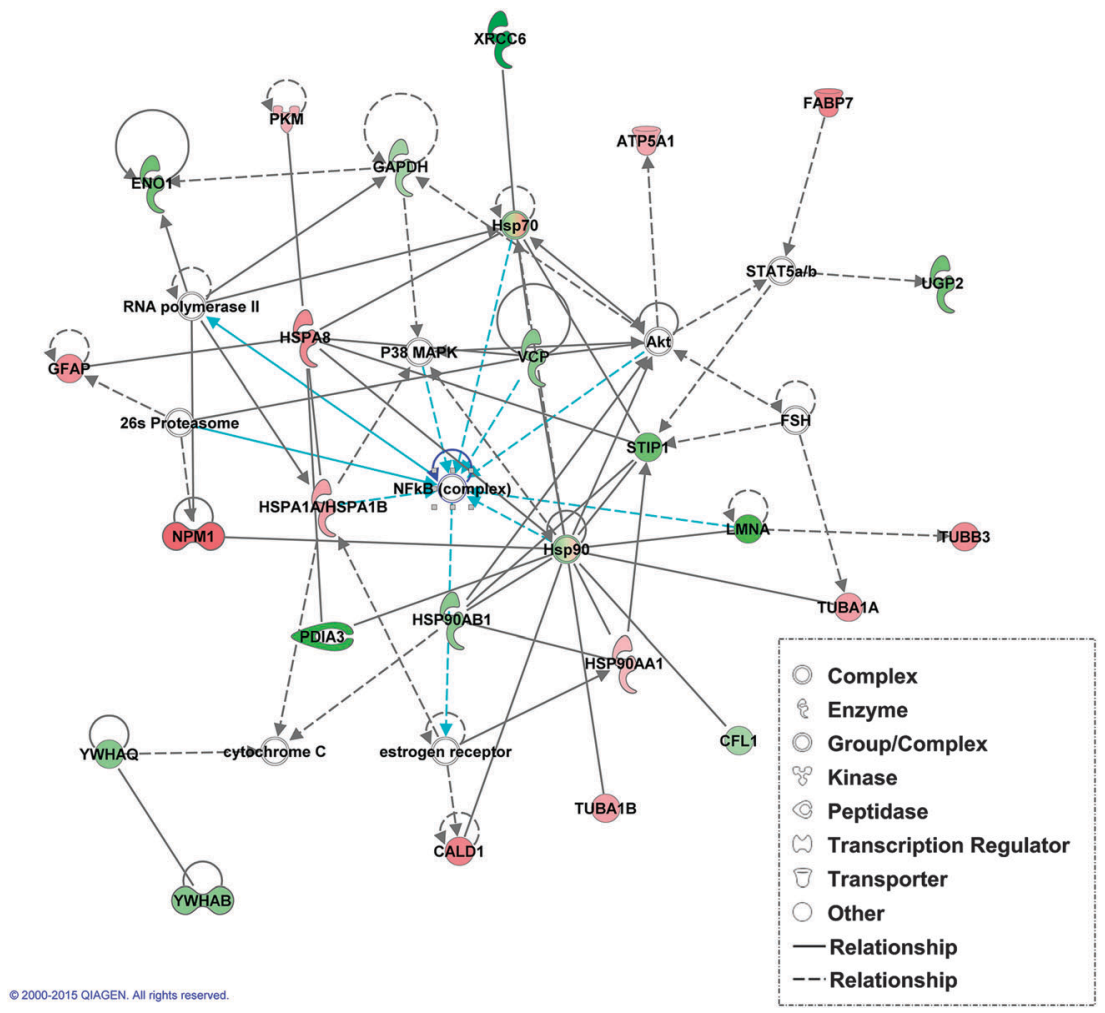

Fig. 3 Graphical representation of the most significant IPA Network (Network \# 2; score $=48$ ). NF- $\kappa B$ complex results in a pivotal position. The connection between Hsp70 and Akt is also evident.

activated NF- $\kappa \mathrm{B}$. We found that IKB $\alpha$ was significantly increased after RA-induced differentiation (Fig. 5). Conversely phosphorylated p65 (P-p65), one of the functional subunits of the NF- $\mathrm{BB}$ complex, was highly expressed in SHH-MB SLCs (Fig. 5) while no change was observed in the expression of total p65 (Fig. 5). These results define the activation of the NF- $\kappa B$ pathway in SLCs and its inhibition after differentiation.

\section{Discussion}

In this study we focused on the proteomic pattern of SHH-MB SLCs and their RA-differentiated counterparts. To perform a differential analysis of the protein repertoire of stem versus RA-treated differentiated MB cells a novel Label Free Differential Proteomic workflow based on the detection of the molecular features was employed.

$\mathrm{MB}$ is an aggressive brain tumor, ${ }^{25}$ which has been stratified into four subgroups. ${ }^{3}$ Among them, we focused on the SHH-driven $\mathrm{MB}$, which is a Hedgehog driven tumor ${ }^{26}$ and also represents a high percentage of $\mathrm{MB}$ with a bimodal age distribution in infants (less than 3 years old) and adult population. ${ }^{27}$ The main purpose of this study was to shed light on new proteins potentially involved in the maintenance and/or regulation of cancer stem like cells derived from such tumors.

The employed peptide-centric differential shotgun profiling was achieved by targeting only the differential signals. This approach can provide an in depth view on the repertoire of less abundant proteins allowing the peptide fragmentation pattern to be matched with a lower false discovery rate. Such a condition is simply provided by the employment of a highly specific MS/MS data dependent acquisition on a limited number of specific differential signals arising from the statistical filtering.

From a total of 994 profiled buckets before and after RA-induced differentiation, we have specifically targeted 554 spectral features from which we identified 68 proteins with significantly altered expression (Tables S1 and S2, ESI $\dagger$ ). We provide evidence of good agreement between MS analysis and western blotting (Fig. 4), thus confirming the quality of the employed antibodies and of the MS data. A large number of proteins significantly increased after differentiation, such as $\alpha$-enolase, a typical marker of neuronal terminally differentiated cells, or GFAP, a marker of glial terminally differentiated cells, underlining the origin of the tumor from primitive embryonic neuroepithelial cells able to differentiate both in neuronal or glial tumor cells. ${ }^{15}$ While others are involved in cytoskeleton remodeling, such as vimentin and $\beta$-actin, or in energy metabolism, such as malate dehydrogenase mitochondrial or triosephosphate isomerase.

Since our aim was to identify proteins characteristic of $\mathrm{SHH}-$ MB SLCs, we mainly focused on proteins, which were downregulated in SLCs after RA-induced differentiation. There are many proteins involved in amino acid metabolism, such as aldolase A; DNA replication, recombination and repair, such as Proliferating Cell Nuclear Antigen (PCNA); and cell-to-cell signaling and interaction, such as protein disulfide-isomerase A3. 
Table 2 IPA results showing the most significantly represented networks and the mainly involved diseases and functions

ID Molecules in network

$1^{a}$ ACTB, Actin, ALB, Alpha tubulin, ANXA6, ATP5J, Beta Tubulin, CCT2, CCT3, CCT5, CCT7, CCT8, DPYSL2, ERK, ERK1/2, FAU, HNRNPC, HNRNPK, HNRNPU, HSPA5, Laminin, LGALS1, MAP2K1/2, Mek, p85(pik3r), PPIA, Ras, Rnr, RPS27A, RPSA, TCP1, TUBB, TUBB4B, VIM, YWHAZ receptor, FABP7, FSH, GAPDH, GFAP, Hsp70, Hsp90, HSP90AA1, HSP90AB1, HSPA8, HSPA1A/HSPA1B, LMNA, NFkB (complex), NPM1, P38 MAPK, PDIA3, PKM, RNA polymerase II, STAT5a/b, STIP1, TUBA1A, TUBA1B, TUBB3, UGP2, VCP, XRCC6, YWHAB, YWHAQ

$3^{a}$ AFP, ALDOA, CCNB2, CD9, CITED1, COTL1, CUL4B, DERL1, EEF2, EEF1A1, ERBB2, FSCN1, GLS, HNRNPU, HSPA9, LAMP2, LDHB, MTBP, MYC, NUDC, OSMR, PDIA6, PFAS, POLD1, PPAT, PRDX1, RNA polymerase I, SET, TNF, TPI1, TUBB2B, TWIST2, VARS, XBP1, YBX3

$4^{a}$ AP2B1, ASL, ATP5B, BCAP31, C2, CDK14, CKB, CLTC, E2F4, GART, GRB2, HINT1, Histone h3, IFNG, KIAA0101, Lh, MDH2, PCM1, PCNA, POLD1, POLH, PPARA, PRKCSH, RFC1, RFC3, RPL10, RPL13, RPL26, RPL32, RRM2B, SNX9, ST13, STAMBP, TOM1, TP53

5 AKT2, APTX, ARRB1, B2M, Collagen Alpha1, Collagen type I, CS, CSF2, DBI, G6PC, Gsk3, H6PD, IDH1, ITGB2, MAPK7, MDH1, MED13, MHC Class II (complex), MSX2, NR3C1, PPARG, PPARGC1B, PRKAR1A, SERPINA1, SFPQ, SLC27A1, SPP1, THRA, TKT, TNFRSF1B, TPM4, TSC22D3, UPP1, VDR, YWHAE

6 DTYMK, MED1

7 PAFAH1B1, PAFAH1B3
2 26s Proteasome, Akt, ATP5A1, CALD1, CFL1, cytochrome C, ENO1, estrogen

Focus

Score molecules Top diseases and functions
24

$48 \quad 2$

$21 \quad 13$

5

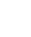

(n)
Cellular assembly and organization, cell-to-cell signaling and interaction, reproductive system development and function

Neurological disease, psychological disorders, cancer

Amino acid metabolism, small molecule biochemistry, cell morphology

DNA replication, recombination, and repair, cancer, neurological disease

Lipid metabolism, Molecular transport, small molecule biochemistry

Nucleic acid metabolism, small molecule biochemistry, organ morphology Lipid metabolism, small molecule biochemistry, cellular development
${ }^{a}$ Networks are connected; the graphical representation is presented in Fig. S3 (ESI).
A

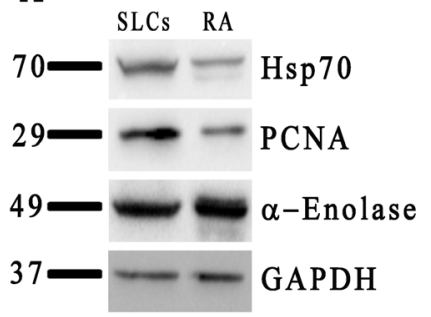

B

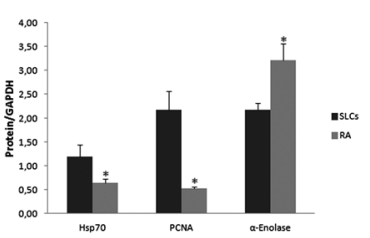

Fig. 4 Confirmation of protein spots by western blot. (A) Western blot analysis of Heat Shock Protein 70 (Hsp70), Proliferating Cell Nuclear Antigen (PCNA) and $\alpha$-enolase in SLCs and in $48 \mathrm{~h}$ RA-treated cells. GAPDH was used as a control for equal protein loads. (B) Densitometric analysis confirmed the significant differential expression observed by proteomic analysis. The protein expression, normalized to GAPDH expression, represents the mean \pm SEM from three independent experiments. * denotes $p<0.05$ versus SLCs.

Among the proteins differentially expressed in SLCs we further investigated Hsp70 (Table S2, ESI, $\dagger$ and Fig. 4) since it is known to correlate with poor prognosis in many cancers, chemotherapy resistance and tumor invasion. ${ }^{28}$ Hsp70 is a molecular chaperone, a member of the heat shock protein family, ${ }^{29}$ whose production is low or undetectable in unstressed, healthy cells, but strongly increased upon a variety of stresses, ${ }^{28}$ such as free radicals, hypoxia as well as high levels of mutant proteins, which are highly present in the tumor microenvironment. ${ }^{30}$ Furthermore, higher expression of Hsp70 has been reported in large cell MBs compared to classic MBs, and were associated with worse prognosis. ${ }^{31}$ Based on our IPA (Fig. 3) and Genomatix analysis (Fig. S4, ESI $\dagger$ ), we found that one of the central regulatory molecules in our network was NF- $\kappa \mathrm{B}$. The NF- $\kappa \mathrm{B}$ complex is
A

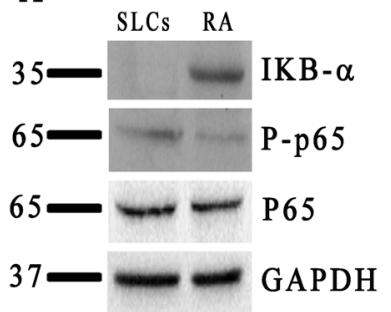

B

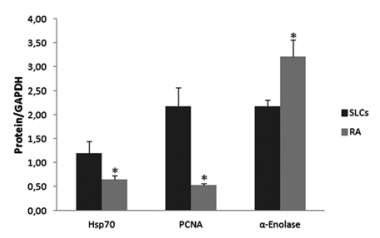

Fig. 5 RA-induced differentiation regulated $N F-\kappa B$ pathway activation. (A) IKB- $\alpha$, P-p65 and p65 expressions were analyzed by means of western blot after 48 h RA-induced cell differentiation. (B) Densitometric analysis showed a significant increase in IKB- $\alpha$ expression, while P-p65 was significantly reduced after RA treatment. Total p65 levels did not change after differentiation. Protein expression was normalized by GAPDH. The data represent mean \pm SEM values from three independent experiments. * denotes $p<0.05$ versus SLCs.

a family of closely related transcription factors involved in the regulation of a wide variety of biological responses, such as immune response and inflammation, cell proliferation and survival and growing evidence also plays a major role in oncogenesis. ${ }^{32}$ By means of western blot analysis we showed that IKB- $\alpha$, the NF- $\kappa \mathrm{B}$ complex main inhibitor, and P-p65, one of the NF- $\kappa \mathrm{B}$ functional subunits, were, respectively, up and down regulated after RA-induced differentiation (Fig. 5), underlining a role of this pathway in SHH-MB SLCs.

Incidentally, a recent study has documented that Foretinib, an orally available multikinase inhibitor, had a dramatic therapeutic effect in SHH-MB, both in vitro and in vivo. ${ }^{17}$ Its activity was due to the inhibition of the MET kinase, a marker of SHH-MB, whose activation triggered multiple downstream effector cascade, 


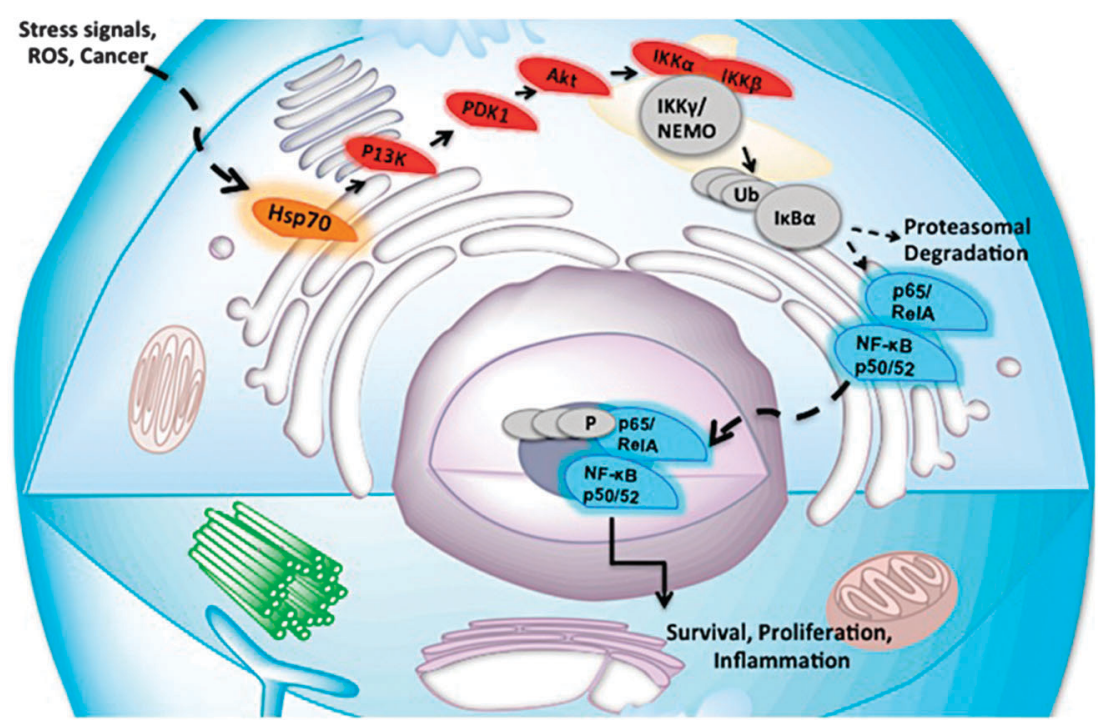

Fig. 6 Hypothesis of the role of Hsp70 in SHH-MB SLCs. A variety of stresses (reactive oxygen species, hypoxia, elevated temperature, altered pH) activate Hsp70. Hsp70 induces Akt, which acts as an upstream regulator of the NF- $\mathrm{kB}$ complex (a classical pathway), leading to p65 phosphorylation and its nuclear translocation and resulting in the deregulation of proliferation and survival.

including PI3K/Akt. ${ }^{17}$ Since Hsp70 promotes tumor cell survival and dissemination ${ }^{28}$ and activates Akt, ${ }^{33}$ an upstream regulator

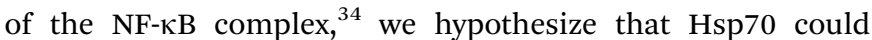
be involved in the biological network maintaining SHH-MB SLCs (Fig. 6).

Taken together, our proteomic results define the human SHH-MB SLC proteins and signalling networks suggesting new key biological players involved in the process of malignancy transmutation.

\section{Experimental procedures}

\section{Materials}

Unless otherwise indicated, media and supplements were purchased from Gibco-Invitrogen (Carlsbad, CA) and chemicals were purchased from Sigma-Aldrich (St. Louis, MO).

\section{Culture of SHH SLCs}

Human infant (less than 3 years old children) medulloblastoma samples (MB) were collected during surgical resection with the approval of institutional review board as described earlier. ${ }^{35}$ Tissues were collected in Hank's Balanced Salt Solution (HBSS) supplemented with $0.5 \%$ glucose and penicillin-streptomycin, grossly triturated with serological pipette and treated with DNAse I to a final concentration of $0.04 \%$ for $20 \mathrm{~min}$. Subsequently, cell aggregates were mechanically disrupted using pipettes of decreasing pore size to obtain a single cell suspension. After dissociation and centrifugation, cells were cultured as neurospheres in selective medium, DMEM/F12 supplemented with $0.6 \%$ glucose, $60 \mathrm{mg} \mathrm{mL}{ }^{-1} N$-acetyl---cysteine, $2 \mathrm{mg} \mathrm{mL}{ }^{-1}$ heparin, $20 \mathrm{ng} \mathrm{mL}^{-1} \mathrm{NGF}, 20 \mathrm{ng} \mathrm{mL}^{-1}$ bFGF (Peprotech, Rocky Hill, NJ), $1 \times$ penicillin-streptomycin and B27 supplement without vitamin A.
For differentiation studies, neurospheres were mechanically dissociated and plated on D-poly-Lysine coated dishes in differentiation medium (DMEM/F12 with N2 supplement and $2 \mathrm{mg} \mathrm{mL}^{-1}$ heparin, $0.6 \%$ glucose, $60 \mathrm{mg} \mathrm{mL}^{-1} N$-acetyl-L-cysteine, containing $1 \%$ fetal bovine serum and RA $8 \mu \mathrm{M}$ ) for $48 \mathrm{~h}$. All samples were prepared in 3 biological replicates for each point.

\section{Protein extraction and digestion}

Cells were lysed in Tris- $\mathrm{HCl} \mathrm{pH}$ 7.6, $50 \mathrm{mM}, \mathrm{NaCl} 150 \mathrm{mM}$, EDTA $1 \mathrm{mM}$, Triton X-100 1\% and a protease inhibitor cocktail (SIGMA P8340). Extracted proteins were precipitated in $80 \%$ acetone, ON at $-20{ }^{\circ} \mathrm{C}$.

Washed protein pellets were subsequently resuspended in a denaturing solution (6 M urea in $100 \mathrm{mM}$ Tris- $\mathrm{HCl} \mathrm{pH}$ 7.8) compatible with trypsin digestion that was conducted as previously described. ${ }^{23}$

\section{Label free data dependent shotgun proteomic analysis}

The samples were diluted with an aqueous solution of $0.1 \% \mathrm{FA}$, $3 \% \mathrm{CH}_{3} \mathrm{CN}$ to a final peptide concentration of $0.4 \mu \mathrm{g} \mu \mathrm{L}^{-1}$ and analyzed by nanoLC-MS on a Proxeon EASY-nLCII (Thermo Fisher Scientific, Milan, Italy) interfaced with a maXis HD UHR-TOF mass spectrometer (Bruker Daltonics $\mathrm{GmbH}$, Bremen, Germany). $2 \mu \mathrm{L}$ of the sample were injected for the MS only runs whereas $5 \mu \mathrm{L}$ for the MS/MS runs. Samples were pre-concentrated on a $\mathrm{C}_{18} \mathrm{-A} 1$ EASY-Column $^{\mathrm{TM}}(2 \mathrm{~cm}, 100 \mu \mathrm{m}$ I.D., $5 \mu \mathrm{m}$ p.s., Thermo Fisher Scientific) for $1 \mathrm{~min}$ at a flow rate of $10 \mu \mathrm{L} \mathrm{min}{ }^{-1}$. Trapped peptides were subsequently separated using a gradient elution on $\mathrm{a} \mathrm{C}_{18}$-Acclaim PepMap $(25 \mathrm{~cm}, 75 \mu \mathrm{m}$ I.D., $5 \mu \mathrm{m}$ p.s., Thermo Fisher Scientific). Flow rate: $0.3 \mu \mathrm{L} \mathrm{min}^{-1}, T 20{ }^{\circ} \mathrm{C}$; eluents: $\mathrm{A}, 0.1 \% \mathrm{FA}$ in $\mathrm{H}_{2} \mathrm{O}$ and $\mathrm{B}, 0.1 \% \mathrm{FA}$ in $\mathrm{CH}_{3} \mathrm{CN}$; gradient: from 2 to $45 \%$ B in $130 \mathrm{~min}$.

Mass spectrometry data were acquired according to the novel label free quantitation workflow developed by Bruker Daltonics. 
Briefly, each sample was first acquired in the MS mode and in triplicate to detect the 'molecular features'. Raw data were processed using the software DataAnalysis v.4.2 to generate a mass list with only exact masses, retention times and intensities. A bucket table was subsequently constructed using ProfileAnalysis v.2.1 (build 268) and a $T$-test performed to highlight the statistically significant differences between the signals detected in each condition. In order to be considered for further processing the molecular features had to satisfy the conditions of a satisfactory replication rate (at least in 4 out of 8 total runs, $50 \%$ and at least in 3 out of 4 replicates, $75 \%$ ) and $p<0.05$ for the relative signal fold change (two-tailed Student's $T$ test). The software automatically generated a list of signals to be included in a scheduled precursor list (SPL) to be targeted in the following MS/MS runs. The samples were then acquired in the MS/MS mode to obtain sequence information of the differential signals. Raw MS/MS data were also processed using DataAnalysis v.4.2 to generate the .xml file for further processing database searching. The quantitation tables together with the MS/MS mass lists were imported in ProteinScape v.3.1.5 474 (Bruker Daltonics GmbH, Bremen, Germany), which was used to perform both protein identifications, protein assessment and linking of the IDs with the bucket tables in order to obtain a list of proteins with the corresponding fold changes. Protein IDs were performed using the MASCOT v.2.4.1 algorithm (http://www.matrixscience.com), against the Uniprot/Swiss-Prot non-redundant database version 2013-08 restricted to Homo sapiens taxonomy (20 266 sequences), setting carbamidomethylation of cysteine as fixed modification and the oxidation of methionine as variable modification, allowing two missed cleavages. A maximal error tolerance of $10 \mathrm{ppm}$ for the precursors and 0.05 Da for the fragment ions were selected according to the high resolution of the mass analyzer. Protein list compilation and assessment were performed using the ProteinExtractor algorithm implemented in ProteinScape setting the following thresholds: the ion score cut-off to 15.0, the Mascot protein scores to $30(p<0.05)$ and the Mascot peptide score to 25 .

In order to identify significant up- or down-regulation the differentially expressed protein dataset was filtered to a threshold of $\pm 20 \%$, hence 1.2 -fold, which is typically 2 times higher than the estimated error in the intensity measurement.

\section{Bioinformatics and statistical analysis}

The Gene Ontology, the KEGG pathway, the Wikipathway and the disease association enrichment analyses were carried out using the Webgestalt online tools (http://bioinfo.vanderbilt. edu/webgestalt/) using the entire Homo sapiens genome as a reference set. Fisher's exact test with the Benjamini \& Hochberg adjustment for multiple comparisons was employed to control the threshold of statistical overrepresentation of biochemical pathways. The 10 pathways with the most significant $p$ values were considered. The list of the differential proteins was also analysed with BioProfiling (http://www.bioprofiling.de/) to obtain the network enrichment, based on known physical protein-protein interactions (IntAct Database). The significant analyses, $p<0.01$, were further considered to interpret and discuss proteomic results. The estimate of the $p$-value provided by the Monte Carlo procedure corresponds to the probability to get a model of the same quality for a random gene list of the same size (random networks statistical environment). Eventually, the enriched network was exported as .xgmml file and visualized and modified by Cytoscape (http://www.cytoscape.org/).

Protein network analysis was performed through the use of QIAGEN's Ingenuity ${ }^{\circledR}$ Pathway Analysis (IPA ${ }^{\circledR}$, QIAGEN Redwood City,www.qiagen.com/ingenuity). IPA constructs hypothetical protein interaction clusters based on the Ingenuity Pathways Knowledge Base. Direct and indirect relationships between the identified proteins were shown as networks on the base of all genes, and endogenous chemicals present in the Ingenuity Knowledge Network scores are calculated as $-\log (p$-value $)$ and indicate the likelihood that focus genes (i.e., the identified proteins within a specific network) are clustered together. Biological functions and canonical pathways over-represented among the identified proteins were also assigned to networks stored in the Ingenuity Pathways Knowledge Base. Biological functions and canonical pathways were ranked in accordance to their significance. Significance was evaluated by an exact Fisher's test.

The list of differentially expressed proteins was used as input for the Genomatix Pathway System (GePS, v.2.7.0, Genomatix Genome Analyzer, v.3.20715). The Median score (Stem, SLCs: Differentiated, RA) was used as a value of expression. Hsp70 was selected and extended once with transcription factors. Based on this first obtained network, NF- $\mathrm{BB}$ (NFKB1) and TP53 were selected and extended once with transcriptional downstream targets producing the final network.

Western blot densitometry data were expressed as mean \pm S.E.M. of at least three independent experiments, each performed in triplicate. Student's $T$ test was performed to evaluate the significance of the differences and experimental data elaborated by means of the GraphPad Prism 5 software (GraphPad Software for Science, San Diego).

\section{Immunochemical analysis}

Cells were lysed in Tris-HCl pH 7.6, $50 \mathrm{mM}$, deoxycholic acid sodium salt 0.5\%, NaCl $140 \mathrm{mM}$, NP40 1\%, EDTA $5 \mathrm{mM}, \mathrm{NaF}$ $100 \mathrm{mM}$, Na pyrophosphate $2 \mathrm{mM}$ and protease inhibitors. For phospho p65, $2 \mathrm{mM}$ Na orthovanadate and $5 \mathrm{mM}$ Na butyrate were also added. Lysates were separated on $8 \%$ or $10 \%$ acrylamide gel and immunoblotted using standard procedures. Rabbit anti-Hsp70, sc-33575 (Santa Cruz Biotechnology, CA), rabbit anti-PCNA, \#13110 (Cell Signaling Technology Inc., Danvers, MA); mouse anti- $\beta 3$-tubulin (TU-20), \#4466 (Cell Signaling Technology Inc, Danvers, MA); mouse anti-GFAP, MAB360 (Merck Millipore, Darmstadt), rabbit anti-p65, \#3034S (Cell Signaling Technology Inc, Danvers, MA); rabbit anti-phospho p65 (P-p65), \#3033S (Cell Signaling Technology Inc, Danvers, MA); mouse anti-GAPDH, ab8245 (AbCam, Cambridge, UK) and HRP-conjugated secondary antisera (Santa Cruz Biotechnology, CA) were used followed by enhanced chemiluminescence (ECL Amersham, Amersham, UK) and images were acquired using the BioRad ChemiDoc MP Imaging System (BioRad, Hercules, CA). Densitometric analysis was performed using the BioRad associated Image Lab Software (BioRad, Hercules, CA). Values are expressed as 
fold over internal control, represented by GAPDH, that does not change significantly in the proteome profiles.

\section{Immunofluorescence analysis}

For immunofluorescence studies, neurospheres were plated on D-poly-lysine coated Lab-Tek chamber slides and allowed to adhere for $3 \mathrm{~h}$. RA-differentiated MB cells (RA) were mechanically dissociated, plated on D-poly-lysine coated Lab-Tek chamber slides and cultured in differentiating medium for 2 days. Cells were fixed with $4 \%$ paraformaldehyde for $10 \mathrm{~min}$ at RT, incubated in permeabilization $(0.1 \%$ Triton $\mathrm{X}-100)$ followed by blocking buffer ( $5 \%$ bovine serum albumin, BSA) and stained overnight with primary antibodies and for $1 \mathrm{~h}$ with secondary antibodies, both diluted in blocking solution. Primary antibodies were mouse anti- $33-T u b u l i n$ (TU-20), \#4466 (Cell Signaling Technology Inc, Danvers, MA) and mouse anti-GFAP, MAB360 (Merck Millipore, Darmstadt). 488-conjugated anti-mouse secondary antibody was purchased from Molecular Probes (Invitrogen, Eugene, OR). Nuclei were counterstained with a Hoechst reagent. Coverslips were mounted with fluorescence mounting medium (S3023, Dako, Carpinteria, CA). Images were acquired using a Carl Zeiss microscope (Axio Observer Z1) using Apotome technology and AxioVision Digital Image Processing Software.

\section{Acknowledgements}

This work was supported by Ministry of University and Research (FIRB RBAP11WCRZ and PRIN), Associazione Italiana per la Ricerca sul Cancro (AIRC), and Istituto Italiano di Tecnologia (IIT).

\section{References}

1 T. A. Dolecek, J. M. Propp, N. E. Stroup and C. Kruchko, Neuro-Oncology, 2012, 14(suppl. 5), v1-v49.

2 U. Tabori, B. Baskin, M. Shago, N. Alon, M. D. Taylor, P. N. Ray, E. Bouffet, D. Malkin and C. Hawkins, J. Clin. Oncol., 2010, 28, 1345-1350.

3 M. D. Taylor, P. A. Northcott, A. Korshunov, M. Remke, Y. J. Cho, S. C. Clifford, C. G. Eberhart, D. W. Parsons, S. Rutkowski, A. Gajjar, D. W. Ellison, P. Lichter, R. J. Gilbertson, S. L. Pomeroy, M. Kool and S. M. Pfister, Acta Neuropathol., 2012, 123, 465-472.

4 T. J. Pugh, S. D. Weeraratne, T. C. Archer, D. A. Pomeranz Krummel, D. Auclair, J. Bochicchio, M. O. Carneiro, S. L. Carter, K. Cibulskis, R. L. Erlich, H. Greulich, M. S. Lawrence, N. J. Lennon, A. McKenna, J. Meldrim, A. H. Ramos, M. G. Ross, C. Russ, E. Shefler, A. Sivachenko, B. Sogoloff, P. Stojanov, P. Tamayo, J. P. Mesirov, V. Amani, N. Teider, S. Sengupta, J. P. Francois, P. A. Northcott, M. D. Taylor, F. Yu, G. R. Crabtree, A. G. Kautzman, S. B. Gabriel, G. Getz, N. Jager, D. T. Jones, P. Lichter, S. M. Pfister, T. M. Roberts, M. Meyerson, S. L. Pomeroy and Y. J. Cho, Nature, 2012, 488, 106-110.

5 P. A. Northcott, D. J. Shih, J. Peacock, L. Garzia, A. S. Morrissy, T. Zichner, A. M. Stutz, A. Korshunov, J. Reimand,
S. E. Schumacher, R. Beroukhim, D. W. Ellison, C. R. Marshall, A. C. Lionel, S. Mack, A. Dubuc, Y. Yao, V. Ramaswamy, B. Luu, A. Rolider, F. M. Cavalli, X. Wang, M. Remke, X. Wu, R. Y. Chiu, A. Chu, E. Chuah, R. D. Corbett, G. R. Hoad, S. D. Jackman, Y. Li, A. Lo, K. L. Mungall, K. M. Nip, J. Q. Qian, A. G. Raymond, N. T. Thiessen, R. J. Varhol, I. Birol, R. A. Moore, A. J. Mungall, R. Holt, D. Kawauchi, M. F. Roussel, M. Kool, D. T. Jones, H. Witt, L. A. Fernandez, A. M. Kenney, R. J. Wechsler-Reya, P. Dirks, T. Aviv, W. A. Grajkowska, M. Perek-Polnik, C. C. Haberler, O. Delattre, S. S. Reynaud, F. F. Doz, S. S. Pernet-Fattet, B. K. Cho, S. K. Kim, K. C. Wang, W. Scheurlen, C. G. Eberhart, M. Fevre-Montange, A. Jouvet, I. F. Pollack, X. Fan, K. M. Muraszko, G. Y. Gillespie, C. Di Rocco, L. Massimi, E. M. Michiels, N. K. Kloosterhof, P. J. French, J. M. Kros, J. M. Olson, R. G. Ellenbogen, K. Zitterbart, L. Kren, R. C. Thompson, M. K. Cooper, B. Lach, R. E. McLendon, D. D. Bigner, A. Fontebasso, S. Albrecht, N. Jabado, J. C. Lindsey, S. Bailey, N. Gupta, W. A. Weiss, L. Bognar, A. Klekner, T. E. Van Meter, T. Kumabe, T. Tominaga, S. K. Elbabaa, J. R. Leonard, J. B. Rubin, L. M. Liau, E. G. Van Meir, M. Fouladi, H. Nakamura, G. Cinalli, M. Garami, P. Hauser, A. G. Saad, A. Iolascon, S. Jung, C. G. Carlotti, R. Vibhakar, Y. S. Ra, S. Robinson, M. Zollo, C. C. Faria, J. A. Chan, M. L. Levy, P. H. Sorensen, M. Meyerson, S. L. Pomeroy, Y. J. Cho, G. D. Bader, U. Tabori, C. E. Hawkins, E. Bouffet, S. W. Scherer, J. T. Rutka, D. Malkin, S. C. Clifford, S. J. Jones, J. O. Korbel, S. M. Pfister, M. A. Marra and M. D. Taylor, Nature, 2012, 488, 49-56.

6 P. A. Northcott, A. Korshunov, H. Witt, T. Hielscher, C. G. Eberhart, S. Mack, E. Bouffet, S. C. Clifford, C. E. Hawkins, P. French, J. T. Rutka, S. Pfister and M. D. Taylor, J. Clin. Oncol., 2011, 29, 1408-1414.

7 P. A. Northcott, A. Korshunov, S. M. Pfister and M. D. Taylor, Nat. Rev. Neurol., 2012, 8, 340-351.

8 P. A. Northcott, D. T. Jones, M. Kool, G. W. Robinson, R. J. Gilbertson, Y. J. Cho, S. L. Pomeroy, A. Korshunov, P. Lichter, M. D. Taylor and S. M. Pfister, Nat. Rev. Cancer, 2012, 12, 818-834.

9 D. T. Jones, N. Jager, M. Kool, T. Zichner, B. Hutter, M. Sultan, Y. J. Cho, T. J. Pugh, V. Hovestadt, A. M. Stutz, T. Rausch, H. J. Warnatz, M. Ryzhova, S. Bender, D. Sturm, S. Pleier, H. Cin, E. Pfaff, L. Sieber, A. Wittmann, M. Remke, H. Witt, S. Hutter, T. Tzaridis, J. Weischenfeldt, B. Raeder, M. Avci, V. Amstislavskiy, M. Zapatka, U. D. Weber, Q. Wang, B. Lasitschka, C. C. Bartholomae, M. Schmidt, C. von Kalle, V. Ast, C. Lawerenz, J. Eils, R. Kabbe, V. Benes, P. van Sluis, J. Koster, R. Volckmann, D. Shih, M. J. Betts, R. B. Russell, S. Coco, G. P. Tonini, U. Schuller, V. Hans, N. Graf, Y. J. Kim, C. Monoranu, W. Roggendorf, A. Unterberg, C. Herold-Mende, T. Milde, A. E. Kulozik, A. von Deimling, O. Witt, E. Maass, J. Rossler, M. Ebinger, M. U. Schuhmann, M. C. Fruhwald, M. Hasselblatt, N. Jabado, S. Rutkowski, A. O. von Bueren, D. Williamson, S. C. Clifford, M. G. McCabe, V. P. Collins, S. Wolf, S. Wiemann, H. Lehrach, 
B. Brors, W. Scheurlen, J. Felsberg, G. Reifenberger, P. A. Northcott, M. D. Taylor, M. Meyerson, S. L. Pomeroy, M. L. Yaspo, J. O. Korbel, A. Korshunov, R. Eils, S. M. Pfister and P. Lichter, Nature, 2012, 488, 100-105.

10 Y. J. Cho, A. Tsherniak, P. Tamayo, S. Santagata, A. Ligon, H. Greulich, R. Berhoukim, V. Amani, L. Goumnerova, C. G. Eberhart, C. C. Lau, J. M. Olson, R. J. Gilbertson, A. Gajjar, O. Delattre, M. Kool, K. Ligon, M. Meyerson, J. P. Mesirov and S. L. Pomeroy, J. Clin. Oncol., 2011, 29, 1424-1430.

11 M. Kool, A. Korshunov, M. Remke, D. T. Jones, M. Schlanstein, P. A. Northcott, Y. J. Cho, J. Koster, A. Schouten-van Meeteren, D. van Vuurden, S. C. Clifford, T. Pietsch, A. O. von Bueren, S. Rutkowski, M. McCabe, V. P. Collins, M. L. Backlund, C. Haberler, F. Bourdeaut, O. Delattre, F. Doz, D. W. Ellison, R. J. Gilbertson, S. L. Pomeroy, M. D. Taylor, P. Lichter and S. M. Pfister, Acta Neuropathol., 2012, 123, 473-484.

12 J. Wang and R. J. Wechsler-Reya, Exp. Neurol., 2014, 260, 69-73.

13 R. J. Wechsler-Reya and M. P. Scott, Neuron, 1999, 22, 103-114. 14 K. E. Ryan and C. Chiang, J. Biol. Chem., 2012, 287, 17905-17913.

15 B. Manoranjan, C. Venugopal, N. McFarlane, B. W. Doble, S. E. Dunn, K. Scheinemann and S. K. Singh, Cancer Lett., 2013, 338, 23-31.

16 M. Kool, D. T. Jones, N. Jager, P. A. Northcott, T. J. Pugh, V. Hovestadt, R. M. Piro, L. A. Esparza, S. L. Markant, M. Remke, T. Milde, F. Bourdeaut, M. Ryzhova, D. Sturm, E. Pfaff, S. Stark, S. Hutter, H. Seker-Cin, P. Johann, S. Bender, C. Schmidt, T. Rausch, D. Shih, J. Reimand, L. Sieber, A. Wittmann, L. Linke, H. Witt, U. D. Weber, M. Zapatka, R. Konig, R. Beroukhim, G. Bergthold, P. van Sluis, R. Volckmann, J. Koster, R. Versteeg, S. Schmidt, S. Wolf, C. Lawerenz, C. C. Bartholomae, C. von Kalle, A. Unterberg, C. Herold-Mende, S. Hofer, A. E. Kulozik, A. von Deimling, W. Scheurlen, J. Felsberg, G. Reifenberger, M. Hasselblatt, J. R. Crawford, G. A. Grant, N. Jabado, A. Perry, C. Cowdrey, S. Croul, G. Zadeh, J. O. Korbel, F. Doz, O. Delattre, G. D. Bader, M. G. McCabe, V. P. Collins, M. W. Kieran, Y. J. Cho, S. L. Pomeroy, O. Witt, B. Brors, M. D. Taylor, U. Schuller, A. Korshunov, R. Eils, R. J. Wechsler-Reya, P. Lichter, S. M. Pfister and I. P. T. Project, Cancer Cell, 2014, 25, 393-405.

17 C. C. Faria, B. J. Golbourn, A. M. Dubuc, M. Remke, R. J. Diaz, S. Agnihotri, A. Luck, N. Sabha, S. Olsen, X. Wu, L. Garzia, V. Ramaswamy, S. C. Mack, X. Wang, M. Leadley, D. Reynaud, L. Ermini, M. Post, P. A. Northcott,
S. M. Pfister, S. E. Croul, M. Kool, A. Korshunov, C. A. Smith, M. D. Taylor and J. T. Rutka, Cancer Res., 2015, 75, 134-146. 18 I. Shats, M. L. Gatza, J. T. Chang, S. Mori, J. Wang, J. Rich and J. R. Nevins, Cancer Res., 2011, 71, 1772-1780.

19 I. Ben-Porath, M. W. Thomson, V. J. Carey, R. Ge, G. W. Bell, A. Regev and R. A. Weinberg, Nat. Genet., 2008, 40, 499-507. 20 A. Mastronuzzi, E. Miele, A. Po, M. Antonelli, F. R. Buttarelli, G. S. Colafati, F. del Bufalo, R. Faedda, G. P. Spinelli, A. Carai, F. Giangaspero, A. Gulino, F. Locatelli and E. Ferretti, BMC Cancer, 2014, 14, 262.

21 F. J. Swartling, M. Cancer, A. Frantz, H. Weishaupt and A. I. Persson, Cell Tissue Res., 2015, 359, 225-254.

22 A. Po, E. Ferretti, E. Miele, E. De Smaele, A. Paganelli, G. Canettieri, S. Coni, L. Di Marcotullio, M. Biffoni, L. Massimi, C. Di Rocco, I. Screpanti and A. Gulino, EMBO J., 2010, 29, 2646-2658.

23 L. Pieroni, F. Finamore, M. Ronci, D. Mattoscio, V. Marzano, S. L. Mortera, S. Quattrucci, G. Federici, M. Romano and A. Urbani, Mol. BioSyst., 2011, 7, 630-639.

24 F. Finamore, L. Pieroni, M. Ronci, V. Marzano, S. L. Mortera, M. Romano, C. Cortese, G. Federici and A. Urbani, Trasfus. Sangue, 2010, 8(suppl. 3), s140-s148.

25 C. Zanini, E. Ercole, G. Mandili, R. Salaroli, A. Poli, C. Renna, V. Papa, G. Cenacchi and M. Forni, PLoS One, 2013, 8, e63748.

26 T. Akino, X. Han, H. Nakayama, B. McNeish, D. Zurakowski, A. Mammoto, M. Klagsbrun and E. Smith, Cancer Res., 2014, 74, 3716-3726.

27 S. E. Leary and J. M. Olson, Curr. Opin. Pediatr., 2012, 24, 33-39.

28 K. Juhasz, A. M. Lipp, B. Nimmervoll, A. Sonnleitner, J. Hesse, T. Haselgruebler and Z. Balogi, Cancers, 2013, 6, 42-66.

29 R. Arya, M. Mallik and S. C. Lakhotia, J. Biosci., 2007, 32, 595-610.

30 K. Xie and S. Huang, Clin. Exp. Metastasis, 2003, 20, 31-43. 31 G. A. Alexiou, G. Vartholomatos, K. Stefanaki, A. Patereli, L. Dova, A. Karamoutsios, G. Lallas, G. Sfakianos, M. Moschovi and N. Prodromou, Pediatrics, 2013, 12, 452-457.

32 X. Dolcet, D. Llobet, J. Pallares and X. Matias-Guiu, Virchows Arch., 2005, 446, 475-482.

33 L. Ding, S. He and X. Sun, Apoptosis, 2014, 19, 1269-1280.

34 X. Gao, Y. Liu, D. Deeb, A. S. Arbab and S. C. Gautam, J. Exp. Ther. Oncol., 2014, 10, 275-283.

35 L. Di Marcotullio, E. Ferretti, A. Greco, E. De Smaele, A. Po, M. A. Sico, M. Alimandi, G. Giannini, M. Maroder, I. Screpanti and A. Gulino, Nat. Cell Biol., 2006, 8, 1415-1423. 